\title{
GLOBAL EXISTENCE AND LIFESPAN FOR SEMILINEAR WAVE EQUATIONS WITH MIXED NONLINEAR TERMS
}

\author{
WEI DAI, DAOYUAN FANG, AND CHENGBO WANG
}

\begin{abstract}
Firstly, we study the equation $\square u=|u|^{q_{c}}+|\partial u|^{p}$ with small data, where $q_{c}$ is the critical power of Strauss conjecture and $p \geq q_{c}$. We obtain the optimal estimate of the lifespan $\ln \left(T_{\varepsilon}\right) \approx \varepsilon^{-q_{c}\left(q_{c}-1\right)}$ in $n=3$, and improve the lower bound of $T_{\varepsilon}$ from $\exp \left(c \varepsilon^{-\left(q_{c}-1\right)}\right)$ to $\exp \left(c \varepsilon^{-\left(q_{c}-1\right)^{2} / 2}\right)$ in $n=2$. Then, we study the Cauchy problem with small initial data for a system of semilinear wave equations $\square u=|v|^{q}, \square v=\left|\partial_{t} u\right|^{p}$ in 3-dimensional space with $q<2$. We obtain that this system admits a global solution above a $p-q$ curve for spherically symmetric data. On the contrary, we get a new region where the solution will blow up.
\end{abstract}

\section{INTRODUCTION}

In this paper, we want to study the global solvability and the blow up for some semilinear wave equations with nonlinear terms like $|u|^{q}$ and $\left|\partial_{t} u\right|^{p}$. Firstly we study the lifespan of the equation with mixed nonlinear terms

$$
\left\{\begin{array}{l}
\square u:=\partial_{t}^{2} u-\Delta u=\left|\partial_{t} u\right|^{p}+|u|^{q}, \\
\left.\left(u, \partial_{t} u\right)\right|_{t=0}=\varepsilon(f(x), g(x)),
\end{array}\right.
$$

where $p>1, q>1$ and $x \in \mathbb{R}^{n}$. This equation is in relation ([9]) with the following equations which are well-investigated:

$$
\begin{gathered}
\square v=|v|^{q}, t>0, x \in \mathbb{R}^{n}, \\
\square w=\left|\partial_{t} w\right|^{p}, t>0, x \in \mathbb{R}^{n} .
\end{gathered}
$$

The first equation (1.2) is related to the Strauss conjecture, for which the critical power, denoted by $q_{c}(n)$, is known to be the positive root of the quadratic equation

$$
(n-1) q^{2}-(n+1) q-2=0 .
$$

This conjecture was finally verified in $[5,18]$. And the complete history can be found in [17]. As for the other equation (1.3), which is related to the Glassey conjecture, see [8] and the references therein for more information.

The global existence and blow up dichotomy for the equation (1.1) with spatial dimension $n=2,3$ has been well understood, through the works $[9,6]$. For the cases where there is no global existence, it is also interesting to give sharp estimates of the lifespan, $T_{\varepsilon}$, from above and below. In [9], the sharp estimates of $T_{\varepsilon}$ has been

Date: April 25, 2019.

2010 Mathematics Subject Classification. 35L05, 35L15, 35L70.

Key words and phrases. Strauss conjecture; Glassey conjecture; generalized Strichartz estimate; Klainerman-Sobolev inequalities. 
obtained for any $p, q \geq 2$ and $q>2 /(n-1)$, except for the critical case $p \geq q=q_{c}$. More precisely, it is known that

$$
\exp \left(c \varepsilon^{-\left(q_{c}-1\right)}\right) \leq T_{\varepsilon} \leq \exp \left(C \varepsilon^{-q_{c}\left(q_{c}-1\right)}\right), p \geq q=q_{c}, n=2,3 .
$$

The lower bound was obtained in [9] by using a variant of Klainerman's method of commuting vector fields, and the upper bound comes from the discussion of Strauss conjecture and a simple application of comparison principle, which is expected to be sharp for this problem.

It is not difficult to find that the lower bound of the lifespan of the critical problem of (1.2) is closely related to the power $q$ of $L_{t}^{q}$ in time norm for the forcing term in the key estimates. For example, the obtained bound $\exp \left(c \varepsilon^{-q_{c}\left(q_{c}-1\right)}\right)$ which comes from [14] coincides with $q=q_{c}$ in estimates (3.1), and the obtained bound $\exp \left(c \varepsilon^{-\left(q_{c}-1\right)^{2} / 2}\right)$ which comes from [11] coincides with $q=\left(q_{c}-1\right) / 2$ in estimates (3.2). In order to improve the result from [9], we adapt these generalized Strichartz estimates to the equation (1.1), use energy inequality with KlainermanSobolev inequality to deal with derivative term. Thus we get the following result for dimension three, which is sharp in general.

Theorem 1.1. Let $n=3, q=q_{c}(3)=1+\sqrt{2}$ and $p \geq q$. Suppose that the data $(f, g)$ satisfy

$$
\Lambda:=\left\|\langle x\rangle^{5} \partial_{x}^{\leq 3} f\right\|_{L_{x}^{\infty}}+\left\|\langle x\rangle^{5} \partial_{x}^{\leq 2} g\right\|_{L_{x}^{\infty}}<\infty,
$$

then there exists an $\varepsilon_{0}(\Lambda, p)>0$ and a constant $c>0$, such that for any $\varepsilon \in\left(0, \varepsilon_{0}\right)$, (1.1) has a unique solution $u \in C^{0}\left(\left[0, \bar{T}_{\varepsilon}\right] ; H^{3}\left(\mathbb{R}^{3}\right)\right) \cap C^{1}\left(\left[0, \bar{T}_{\varepsilon}\right] ; H^{2}\left(\mathbb{R}^{3}\right)\right)$ where

$$
\bar{T}_{\varepsilon}:=\exp \left(c \varepsilon^{-q_{c}\left(q_{c}-1\right)}\right) .
$$

Turning to the 2 dimensional case, as is typical for wave equations, the problem seems to be more delicate. As far as the authors are aware, even for the problem (1.2) with $q=q_{c}(2)$, the only available approach to prove the sharp lower bound is given in [19], for compactly supported smooth data, which relies heavily on the fundamental solutions and seems inappropriate for the problems involving nonlinear term $\left|\partial_{t} u\right|^{p}$. Instead, the approach using space-time estimates is more robust for various nonlinear problems. Here, we adapt the generalized Strichartz estimates from [11] to the current setting and obtain the following

Theorem 1.2. Let $n=2, q=q_{c}(2)=(3+\sqrt{17}) / 2$ and $p \geq q$. Considering (1.1) with data $(f, g)$ satisfying (1.4), we have a similar existence result with small data, as in Theorem 1.1, with $\bar{T}_{\varepsilon}$ replaced by

$$
\tilde{T}_{\varepsilon}:=\exp \left(c \varepsilon^{-\left(q_{c}-1\right)^{2} / 2}\right) .
$$

Next, we consider a coupled wave system with different nonlinear terms in each equation,

$$
\left\{\begin{array}{l}
\square u=|v|^{q}, \quad \square v=\left|\partial_{t} u\right|^{p}, \\
\left.\left(u, \partial_{t} u\right)\right|_{t=0}=\varepsilon(f(x), g(x)),\left.\left(v, \partial_{t} v\right)\right|_{t=0}=\varepsilon(\tilde{f}(x), \tilde{g}(x)),
\end{array}\right.
$$

where $u, v$ depend on $(t, x) \in[0, T) \times \mathbb{R}^{3}$ for some $T \in(0, \infty], \varepsilon$ is positive and small enough. 
The system (1.5) has been discussed in [10], which shows there exists a curve in $(1, \infty)^{2}$ of index-pairs $(p, q)$,

$$
(p-1)(p q-1)=p+2, \quad 1<q, \quad 1<p<3 .
$$

When $(p, q)$ lies below the curve, this system blow up in most cases whatever small $\varepsilon$ is. On the contrary, when $(p, q)$ lies above the curve with $2<p<3,2<q$, this system (1.5) has a global solution at least for radially symmetric small data.

To analyze this equation, we compare it with some closely related systems. They are

$$
\begin{aligned}
& \text { (I) : } \square u=|v|^{q}, \quad \square v=|u|^{p}, \quad x \in \mathbb{R}^{3} \text {; } \\
& \text { (II) : } \square u=\left|\partial_{t} v\right|^{q}, \quad \square v=\left|\partial_{t} u\right|^{p}, \quad x \in \mathbb{R}^{3} \text {. }
\end{aligned}
$$

For (I), which relates to the Strauss conjecture, it is known that

$$
\max \left\{\frac{p+2+q^{-1}}{p q-1}, \frac{q+2+p^{-1}}{p q-1}\right\}=1
$$

is the critical curve of index-pairs $(p, q)$. The curve was firstly provided in [2], in which they prove global existence for supercritical case and blow up for subcritical case. For blow up in the critical case and the lifespan estimates, see [1] and the references therein.

As for (II), which relates to the Glassey conjecture, such a curve is

$$
\max \left\{\frac{q+1}{p q-1}, \frac{p+1}{p q-1}\right\}=1 .
$$

This is optimal at least for radially symmetric initial data, where the blow up part can be found in [3] and the existence part for symmetric situation was verified in [13]. We refer the interested readers to [10] for more information about these two problems.

It is naturally to infer that the critical curve for (1.5) should lies between the curves (1.7) and (1.8). However, the curve (1.6) intersects with one of the above curves (see the figure below, $C C^{\prime \prime}$ intersects with $l_{B}$ ). This motivates us to improve the result when $q<2$.

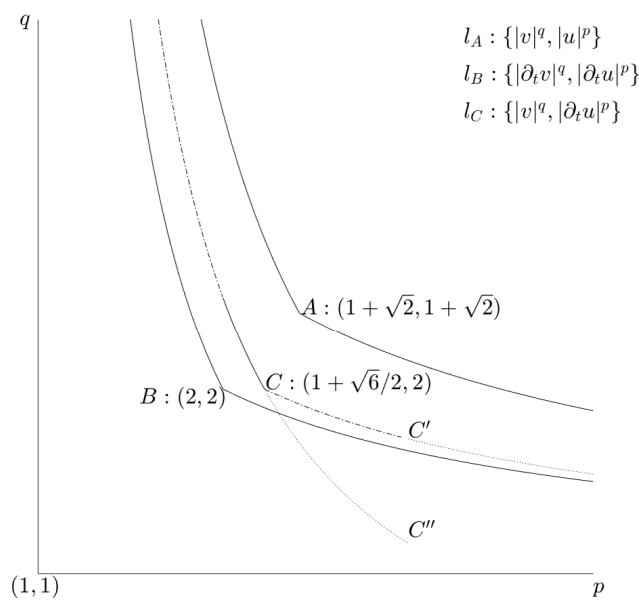


Here $l_{A}$ is the critical curve to problem $(\mathrm{I}), l_{B}$ is the critical curve to problem (II) and $C C^{\prime \prime}$ is (1.6) with $q<2$. We want to establish a global existence theorem to (1.5) with radially symmetric small data, for the region above $C C^{\prime}$,

$$
p(q-1)>2+\frac{1}{p q}, \quad 1<q<2, \quad 2<p<3 .
$$

Theorem 1.3. Suppose that (1.9) is satisfied. We also suppose $f, \tilde{f} \in C^{2}\left(\mathbb{R}^{3}\right)$, $g, \tilde{g} \in C^{1}\left(\mathbb{R}^{3}\right)$ are spherically symmetric and supported in $B_{1}(0)$. Then there exists a positive number $\varepsilon_{0}$ such that for $0<\varepsilon<\varepsilon_{0}$, there exists a global solution $(u, v)$ of (1.5) satisfying

$$
u, v, \partial_{t} u \in C\left(\mathbb{R}^{+} \times \mathbb{R}^{3}\right) .
$$

The key point here is that a symmetric 3-D wave equation is equivalent to an 1-D equation essentially, in which case the solution of linear problem has a higher regularity. In order to match the situation $q<2$, we use the weight functions different from [10].

As for the blow up part, we adopt the strategy of deriving a system of ordinary differential inequalities which causes blow up solutions. Since the technique is suitable for any dimensions, we give a general result rather than $n=3$.

Theorem 1.4. Let $n \geq 1$ and $p, q>1$ such that

$$
\frac{p+1}{p q-1}>\frac{n-1}{2}, \quad \frac{n-1}{2}(q-1)<1 .
$$

Suppose that all data are supported in $B_{1}(0)$ and $g-f, f, \tilde{g}-\tilde{f}, \tilde{f}$ are non-negative where $\tilde{f}$ does not vanish identically. Then for any $\varepsilon>0$, there are no global weak solutions of (1.5) such that $\operatorname{supp}(u, v) \subset\{(t, x):|x| \leq t+1\}$, and

$$
u, v, \partial_{t} u, \partial_{t} v \in C\left(\mathbb{R}_{+} ; L^{1}\left(\mathbb{R}^{n}\right)\right), v \in C\left(\mathbb{R}_{+} ; L^{q}\left(\mathbb{R}^{n}\right)\right), \partial_{t} u \in C\left(\mathbb{R}_{+} ; L^{p}\left(\mathbb{R}^{n}\right)\right),
$$

where $\mathbb{R}_{+}=[0, \infty)$.

This improves the result from the curve $C C^{\prime \prime}$ to the right branch of $l_{B}$. Remark also that this result is better than that in [15].

Remark 1.5. For $n=3$, partly because the endpoint $(p, q)=(\sqrt{6} / 2+1,2)$ is critical, but $(p, q)=(2,2)$ on $l_{B}$ is not, we tend to believe that the critical curve for (1.5) with $q \in(1,2)$ is that appeared in Theorem 1.3 , that is,

$$
p(q-1)=2+\frac{1}{p q}, \quad 1<q<2, \quad 1<p
$$

which is $C C^{\prime}$ in the figure.

This rest of paper is organized as follows. In Section 2 we introduce some notations we will use. Then we prove Theorem 1.1-1.4, in Section 3-5, respectively.

\section{NOTATIONS}

We list here some notations which will be used in our article. First, the Einstein summation convention is used, as well as the convention that Greek indices $\mu, \nu, \cdots$ range from 0 to $n$ while Latin indices $i, j, \cdots$ will run from 1 to $n$. 
We also denote

$$
\begin{aligned}
& \langle a\rangle:=\sqrt{1+|a|^{2}} \approx 1+|a|, \\
& \|f(x)\|_{\mathcal{L}_{r}^{p} L_{\omega}^{b}}:=\left(\int_{0}^{\infty}\left(\int_{S^{n-1}}|f(r \omega)|^{b} \mathrm{~d} \omega\right)^{p / b} r^{n-1} \mathrm{~d} r\right)^{1 / p}, \\
& \partial u(t, x):=\left\{\partial_{\mu} u\right\}=\left(\partial_{t} u, \partial_{x} u\right), \\
& \partial^{\leq k} f:=\left\{\partial^{\alpha} f\right\}_{0 \leq|\alpha| \leq k} .
\end{aligned}
$$

Furthermore, we will use some kinds of special vector fields. There are spatial rotation: $\Omega_{j k}=x^{k} \partial_{j}-x^{j} \partial_{k}$, Lorentz boost: $\Omega_{0 k}=t \partial_{k}+x^{k} \partial_{t}$ and scaling: $L_{0}=$ $t \partial_{t}+x^{i} \partial_{i}$. Set $\Gamma=\left\{\partial_{\mu}, \Omega_{\mu \nu}, L_{0}\right\}$ be the well-known Klainerman vector fields. For such vector fields, we have

$$
\begin{aligned}
& {\left[\partial_{\mu}, \Gamma_{\alpha}\right] f=C_{\mu \alpha}^{\beta} \partial_{\beta} f, \quad\left[\Gamma_{\alpha}, \Gamma_{\beta}\right] f=C_{\alpha \beta}^{\sigma} \Gamma_{\sigma} f,} \\
& {\left[\Gamma_{\alpha}, \square\right] f=C_{\alpha} \square f, \quad\left(\alpha, \beta=0,1, \cdots,\left(n^{2}+3 n+2\right) / 2\right)}
\end{aligned}
$$

where $[X, Y]$ denotes the commutator $X Y-Y X$. And all coefficients are belong to $C_{b}^{\infty}$

We denote a constant $C$ which may change from line to line, but not depend on $\varepsilon, t$ or $x$. And $A \lesssim B$ means $A \leq C B$ for some $C>0, A \gtrsim B$ is similar, $A \approx B$ means $A \lesssim B \lesssim A$.

\section{Proof of Theorem 1.1 And Theorem 1.2}

\subsection{Preliminaries.}

We firstly list some lemmas to be used later.

Lemma 3.1 (Local existence). When $n \leq 3$. For the equation

$$
\left\{\begin{array}{l}
\partial_{t}^{2} u-\Delta u=F(u, \partial u), \\
u(0, x)=f(x), u_{t}(0, x)=g(x) .
\end{array}\right.
$$

If $(f, g) \in H^{3} \times H^{2}, F \in C^{2}$ and $F(0,0)=0$ then there is a $T>0$, depending on the norm of the data, so that this Cauchy problem has a unique solution satisfying

$$
\left\|\partial^{\leq 3} u(t, \cdot)\right\|_{L_{x}^{2}}<\infty, \quad 0 \leq t \leq T
$$

Also, if $T_{*}$ is the supremum over all such times $T$, then either $T_{*}=\infty$ or

$$
|\partial \leq 3 u| \notin L_{t}^{\infty} L_{x}^{2}\left(0 \leq t<T_{*}\right) .
$$

Proof. The result is classical, for a proof, see, e.g., Chapter 12 of [4].

Lemma 3.2 (Theorem 6.4 of [14]). When $n=3$, suppose $u$ solves the linear equation

$$
\left\{\begin{array}{l}
\square u(t, x)=F(t, x), \\
u(0, x)=f(x), u_{t}(0, x)=g(x)
\end{array}\right.
$$


in $\left[0, T_{*}\right) \times \mathbb{R}^{3}$. Then there exists a constant $C$, such that $\forall T<T_{*}$

$$
\begin{aligned}
T^{1 / q_{c}^{2}}\|u(T, \cdot)\|_{\mathcal{L}_{r}^{q_{c}} L_{\omega}^{3} \leq} & C\left(\left\|r^{1-2 / q_{c}} \partial_{\omega}^{\leq 1} f\right\|_{\mathcal{L}_{r}^{q_{c}} L_{\omega}^{3 / 2}}+\left\|r^{1+1 / q_{c}} \partial_{\omega}^{\leq 1} f\right\|_{\mathcal{L}_{r}^{\infty} L_{\omega}^{3 / 2}}\right. \\
& +\left\|r^{2-2 / q_{c}} g\right\|_{\mathcal{L}_{r}^{q_{c}} L_{\omega}^{3 / 2}}+\left\|r^{2+1 / q_{c}} g\right\|_{\mathcal{L}_{r}^{\infty} L_{\omega}^{3 / 2}} \\
& \left.+\|F\|_{L_{t}^{q_{c}} \mathcal{L}_{r}^{1} L_{\omega}^{3 / 2}(t<T / 4)}+T^{1 / q_{c}}\|F\|_{L_{t}^{\infty} \mathcal{L}_{r}^{1} L_{\omega}^{3 / 2}(T / 4<t<T)}\right) .
\end{aligned}
$$

Lemma 3.3 (Theorem 1.4 of [11]). When $n=2$, suppose $u$ solves the linear equation

$$
\left\{\begin{array}{l}
\square u(t, x)=F(t, x), \\
u(0, x)=f(x), u_{t}(0, x)=g(x)
\end{array}\right.
$$

in $\left[0, T_{*}\right) \times \mathbb{R}^{2}$. Let $q_{*}=\frac{q_{c}-1}{2}, s_{c}=1-1 / q, b>\frac{1}{q_{*} q_{c}}+\frac{1}{q_{c}}, X^{b} \equiv H_{\omega}^{s_{c}+\delta, b} \times$ $H_{\omega}^{s_{c}-1+\delta, b}(\delta>0)$, where

$$
H_{\omega}^{s, b}=\left\{u \in H^{s}:\left\|\left(1-\Delta_{\omega}\right)^{b / 2} u\right\|_{H^{s}}<\infty\right\}, \quad \Delta_{\omega}=\sum_{1 \leq i<j \leq n} \Omega_{i j}^{2} .
$$

Then there exists a constant $C$, such that $\forall T<T_{*}$

$$
\|u\|_{L_{t}^{q_{*} q_{c}} \mathcal{L}_{r}^{q_{c}} L_{\omega}^{2}(t<T)} \leq C(\ln (2+T))^{1 /\left(q_{*} q_{c}\right)}\left(\|(f, g)\|_{X^{b}}+\|F\|_{L_{t}^{q_{*}} \mathcal{L}_{r}^{1} L_{\omega}^{2}}\right) .
$$

Lemma 3.4 (Energy inequality). For any $n$, suppose $u$ solves the linear equation

$$
\left\{\begin{array}{l}
\square u(t, x)=F(t, x), \\
u(0, x)=f(x), u_{t}(0, x)=g(x) .
\end{array}\right.
$$

We have, $\forall T>0$

$$
\|\partial u(T, \cdot)\|_{L_{x}^{2}} \leq\|\partial u(0, \cdot)\|_{L_{x}^{2}}+\|F\|_{L_{t}^{1} L_{x}^{2}(t<T)}
$$

Since the property of commutator (2.1), we also have for any $k \in \mathbb{Z}^{+}$

$$
\left\|\partial \Gamma^{\leq k} u(T, \cdot)\right\|_{L_{x}^{2}} \leq C_{k}\left(\left\|\partial \Gamma^{\leq k} u(0, \cdot)\right\|_{L_{x}^{2}}+\left\|\Gamma^{\leq k} F\right\|_{L_{t}^{1} L_{x}^{2}(t<T)}\right) .
$$

Lemma 3.5 (Klainerman-Sobolev inequalities). Let $v \in C^{\infty}\left(\mathbb{R}^{1+n}\right)$ vanish when $|x|$ is large, $1 \leq p<\infty$ and $k>n / p$, then if $t>0$

$$
\langle t+r\rangle^{(n-1) / p}\langle t-r\rangle^{1 / p}|v(t, x)| \leq C\left\|\Gamma^{\leq k} v(t, \cdot)\right\|_{L_{x}^{p}} .
$$

Otherwise if $1 \leq p<q<\infty$ and $k \geq n / p-n / q$, then

$$
\langle t\rangle^{(n-1)(1 / p-1 / q)}\|v(t, \cdot)\|_{L_{x}^{q}} \leq C\left\|\Gamma^{\leq k} v(t, \cdot)\right\|_{L_{x}^{p}} .
$$

Proof. The inequality (3.3) is the well-known Klainerman-Sobolev inequality, which is proved in [12]. Heuristically, the inequality (3.4) can be viewed as a consequence of (3.3). We refer $[7,(3.9)]$ for a complete proof.

Lemma 3.6 (Sobolev embedding). Let $x \in \mathbb{R}^{n}$, and $1 \leq p<q \leq \infty$, then

$$
\|f(x)\|_{L_{x}^{q}} \leq C\left\|\partial^{\leq k} f(x)\right\|_{L_{x}^{p}},
$$

where $k \geq n / p-n / q$ if $q<\infty$, or $k>n / p$ if $q=\infty$. When it comes to $S^{n-1}$, with $1 \leq p<q \leq \infty$, then

$$
\|f(\omega)\|_{L_{\omega}^{p}} \leq C\left\|\Omega^{\leq k} f(\omega)\right\|_{L_{\omega}^{p}},
$$


where $k \geq(n-1) / p-(n-1) / q$ if $q<\infty$, or $k>(n-1) / p-(n-1) / q$ if $q=\infty$. For mixed-norm, we have

$$
\|f(x)\|_{\mathcal{L}_{r}^{q} L_{\omega}^{4}} \leq C\left\|\partial^{\leq k} f(x)\right\|_{\mathcal{L}_{r}^{p} L_{\omega}^{2}},
$$

where $2 \leq p \leq q \leq 4$ and $k \geq n / 4$.

Proof. Here inequality (3.5) is known as Sobolev embedding, and inequality (3.6) just the same inequality on sphere. Finally the inequality (3.7) is a simple Corollary of lemma 3.2 in [16] and normal Sobolev embedding in a small ball contains origin point.

Claim 3.7. We claim that there exists a constant $C_{0}$ depends on $\Lambda, n$ but not on $\varepsilon$ if $\varepsilon$ is small enough, then all of the initial norms we will use can be bounded by $C_{0} \varepsilon$.

The proof of Claim 3.7 need some delicate calculation, so we will postpone it to the end of this section. For now, we are ready to prove the Theorem 1.1 and Theorem 1.2.

\subsection{Proof of Theorem $1.1(n=3)$.}

By Lemma 3.1, we only need to prove that if $T_{\varepsilon} \leq \bar{T}_{\varepsilon}$, then $\left\|\partial^{\leq 3} u\right\|_{L_{t}^{\infty} L_{x}^{2}\left(t<T_{\varepsilon}\right)} \leq$ $M<\infty$, which gives the contradiction.

Firstly, we want to prove that for some suitable $C_{M}, c, \varepsilon_{0}$ to be fixed later, if $0 \leq T<T_{\varepsilon} \leq \bar{T}_{\varepsilon}$ then

$$
\begin{array}{ll}
A(T)=\langle T\rangle^{1 / q_{c}^{2}}\left\|\Gamma^{\leq 2} u(T, \cdot)\right\|_{\mathcal{L}_{r}^{q_{c}} L_{\omega}^{3}} & \leq C_{M} \varepsilon, \\
B(T)=\left\|\partial \Gamma^{\leq 2} u(T, \cdot)\right\|_{L_{x}^{2}} & \leq C_{M} \varepsilon .
\end{array}
$$

With the help of Claim 3.7, we choose $C_{M}$ satisfying that $A(0), B(0) \leq C_{M} \varepsilon / 4$. Take $X=\left\{T \in\left[0, T_{\varepsilon}\right): A(t), B(t) \leq C_{M} \varepsilon, \forall t \in[0, T]\right\}$. We want to prove $X=$ $\left[0, T_{\varepsilon}\right)$. Consequently, it would suffice to show that, if $T$ is as above, then equation (3.8) implies that $A(T), B(T) \leq C_{M} \varepsilon / 2$. Because of Lemma 3.1, we assume $T>1$ without loss of generality.

Part 1: Estimate of $A(t)$.

By Lemma 3.2, we obtain

$$
\begin{aligned}
A(T) \leq & C_{M} \varepsilon / 4+C\left\|\Gamma^{\leq 2}|u|^{q_{c}}\right\|_{L_{t}^{q_{c}} \mathcal{L}_{r}^{1} L_{\omega}^{3 / 2}(t<T / 4)} \\
& +C\left\|\Gamma^{\leq 2}|\partial u|^{p}\right\|_{L_{t}^{q_{c}} \mathcal{L}_{r}^{1} L_{\omega}^{3 / 2}(t<T / 4)} \\
& +C T^{1 / q_{c}}\left\|\Gamma^{\leq 2}|u|^{q_{c}}\right\|_{L_{t}^{\infty} \mathcal{L}_{r}^{1} L_{\omega}^{3 / 2}(T / 4<t<T)} \\
& +C T^{1 / q_{c}}\left\|\Gamma^{\leq 2}|\partial u|^{p}\right\|_{L_{t}^{\infty} \mathcal{L}_{r}^{1} L_{\omega}^{3 / 2}(T / 4<t<T)} .
\end{aligned}
$$


First we consider the spatial norm part of $|u|$ terms, by Hölder's inequality and Lemma 3.6, we see

$$
\begin{aligned}
\left\|\Gamma^{\leq 2}|u|^{q_{c}}\right\|_{\mathcal{L}_{r}^{1} L_{\omega}^{3 / 2}} & \leq C\left\|\left|\Gamma^{\leq 2} u\left\|\left.u\right|^{q_{c}-1}\right\|_{\mathcal{L}_{r}^{1} L_{\omega}^{3 / 2}}+C\left\|\left|\Gamma^{\leq 1} u\right|^{2}|u|^{q_{c}-2}\right\|_{\mathcal{L}_{r}^{1} L_{\omega}^{3 / 2}}\right.\right. \\
& \leq C\left\|\Gamma^{\leq 2} u\right\|_{\mathcal{L}_{r}^{q_{c}} L_{\omega}^{3}}\|u\|_{\mathcal{L}_{r}^{q_{c}} L_{\omega}^{3 \sqrt{2}}}^{q_{c}}+C\left\|\Gamma^{\leq 1} u\right\|_{\mathcal{L}_{r}^{q_{c}} L_{\omega}^{3}}^{2}\|u\|_{\mathcal{L}_{r}^{q_{c}} L_{\omega}^{\infty}}^{q_{\omega}-2} \\
& \leq C\left\|\Gamma^{\leq 2} u\right\|_{\mathcal{L}_{r}^{q_{c}} L_{\omega}^{3}}^{q_{c}} \\
& \leq C\left(\langle t\rangle^{-1 / q_{c}^{2}} A(t)\right)^{q_{c}} \\
& \leq C C_{M}^{q_{c}} \varepsilon^{q_{c}}\langle t\rangle^{-1 / q_{c}}
\end{aligned}
$$

Then, we consider the spatial norm part of $|\partial u|$ terms, by Hölder's inequality and Lemma 3.5, Lemma 3.6, we have

$$
\begin{aligned}
\left\|\Gamma^{\leq 2}|\partial u|^{p}\right\|_{\mathcal{L}_{r}^{1} L_{\omega}^{3 / 2}} & \leq C\left\|\left|\Gamma^{\leq 2} \partial u\left\|\left.\partial u\right|^{p-1}\right\|_{\mathcal{L}_{r}^{1} L_{\omega}^{3 / 2}}+C\left\|\left|\Gamma^{\leq 1} \partial u\right|^{2}|\partial u|^{p-2}\right\|_{\mathcal{L}_{r}^{1} L_{\omega}^{3 / 2}}\right.\right. \\
& \leq C\left\|\Gamma^{\leq 2} \partial u\right\|_{\mathcal{L}_{r}^{2} L_{\omega}^{2}}\|\partial u\|_{\mathcal{L}_{r}^{2} L_{\omega}^{6}}\|\partial u\|_{\mathcal{L}_{r}^{\infty} L_{\omega}^{\infty}}^{p-2}+C\left\|\Gamma^{\leq 1} \partial u\right\|_{\mathcal{L}_{r}^{2} L_{\omega}^{3}}^{2}\|\partial u\|_{\mathcal{L}_{r}^{\infty} L_{\omega}^{\infty}}^{p-2} \\
& \leq C\left\|\Gamma^{\leq 2} \partial u\right\|_{L_{x}^{2}}^{2}\|\partial u\|_{L_{x}^{\infty}}^{p-2} \\
& \leq C\left\|\Gamma^{\leq 2} \partial u\right\|_{L_{x}^{2}}^{p}\left(\langle t\rangle^{-1}\right)^{p-2} \\
& \leq C C_{M}^{p} \varepsilon^{p}\langle t\rangle^{2-p}
\end{aligned}
$$

Back to equation (3.9), assume $\varepsilon_{0}<1 / C_{M}$. Since $p \geq q_{c}=1+\sqrt{2}, 0<\varepsilon<\varepsilon_{0}$, we conclude

$$
\begin{aligned}
A(T) \leq & C_{M} \varepsilon / 4+C C_{M}^{q_{c}} \varepsilon^{q_{c}}\left(1+\left(\int_{0}^{T / 4}\langle t\rangle^{-1} \mathrm{~d} t\right)^{1 / q_{c}}\right) \\
& +C C_{M}^{p} \varepsilon^{p}\left(\langle T\rangle^{q_{c}-p}+\left(\int_{0}^{T / 4}\langle t\rangle^{-q_{c}(p-2)} \mathrm{d} t\right)^{1 / q_{c}}\right) \\
\leq & C_{M} \varepsilon\left(1 / 4+C C_{M}^{q_{c}-1} \varepsilon^{q_{c}-1}(\ln (T+2))^{1 / q_{c}}+C C_{M}^{p-1} \varepsilon^{p-1}\right) \\
\leq & C_{M} \varepsilon\left(1 / 3+C C_{M}^{q_{c}-1} c^{1 / q_{c}}+C C_{M}^{q_{c}-1} \varepsilon_{0}^{q_{c}-1}\right) .
\end{aligned}
$$

Part 2: Estimate of $B(t)$.

For this part, by the Lemma 3.4, we know that

$$
B(T) \leq C_{M} \varepsilon / 4+C\left\|\Gamma^{\leq 2}|u|^{q_{c}}\right\|_{L_{t}^{1} L_{x}^{2}(t<T)}+C\left\|\Gamma^{\leq 2}|\partial u|^{p}\right\|_{L_{t}^{1} L_{x}^{2}(t<T)} .
$$

For the $|u|$ term, by Hölder's inequality and Lemma 3.5, we have

$$
\begin{aligned}
\left\|\Gamma^{\leq 2}|u|^{q_{c}}\right\|_{L_{x}^{2}} & \leq C\left\|\Gamma^{\leq 2} u\right\|_{L_{x}^{q_{c}}}\|u\|_{L_{x}^{a}}^{q_{c}-1}+C\left\|\Gamma^{\leq 1} u\right\|_{L_{x}^{4}}^{2}\|u\|_{L_{x}^{\infty}}^{q_{c}-2} \\
& \leq C\left\|\Gamma^{\leq 2} u\right\|_{L_{x}^{q_{c}}}^{q_{c}}\left(\langle t\rangle^{-2\left(1 / q_{c}-1 / a\right)\left(q_{c}-1\right)}+\langle t\rangle^{-2\left(1 / q_{c}-1 / 4\right) \cdot 2-2\left(1 / q_{c}\right)\left(q_{c}-2\right)}\right) \\
& \leq C\left\|\Gamma^{\leq 2} u\right\|_{L_{x}^{q_{c}}}^{q_{c}}\langle t\rangle^{-1} \\
& \leq C\left(\langle t\rangle^{-1 / q_{c}^{2}} A(t)\right)^{q_{c}}\langle t\rangle^{-1} \\
& \leq C C_{M}^{q_{c}} \varepsilon^{q_{c}}\langle t\rangle^{1-q_{c}},
\end{aligned}
$$


where $a=\left(q_{c}-1\right) /\left(1 / 2-1 / q_{c}\right)$.

For the $|\partial u|$ term, by Hölder's inequality and Lemma 3.5 again, we know

$$
\begin{aligned}
\left\|\Gamma^{\leq 2}|\partial u|^{p}\right\|_{L_{x}^{2}} & \leq C\left\|\Gamma^{\leq 2} \partial u\right\|_{L_{x}^{2}}\|\partial u\|_{L_{x}^{\infty}}^{p-1}+C\left\|\Gamma^{\leq 1} \partial u\right\|_{L_{x}^{4}}^{2}\|\partial u\|_{L_{x}^{\infty}}^{p-2} \\
& \leq C\left\|\Gamma^{\leq 2} \partial u\right\|_{L_{x}^{2}}^{p}\left(\langle t\rangle^{-2(1 / 2)(p-1)}+\langle t\rangle^{-2(1 / 2-1 / 4) 2-2(1 / 2)(p-2)}\right) \\
& \leq C C_{M}^{p} \varepsilon^{p}\langle t\rangle^{1-p} .
\end{aligned}
$$

Back to (3.10), we obtain

$$
B(T) \leq C_{M} \varepsilon\left(1 / 4+C C_{M}^{q_{c}-1} \varepsilon^{q_{c}-1}+C C_{M}^{p-1} \varepsilon^{p-1}\right) \leq C_{M} \varepsilon\left(1 / 4+C C_{M}^{q_{c}-1} \varepsilon_{0}^{q_{c}-1}\right) .
$$

Part 3: The boundness of $A(t), B(t)$.

We choose $\varepsilon_{0}$ and the constant $c$ in $\bar{T}_{\varepsilon}$ small enough, such that $A(T), B(T) \leq$ $C_{M} \varepsilon / 2$, which completes the proof.

\subsection{Proof of Theorem 1.2( $n=2)$.}

Similar to last subsection, we need to prove that for some suitable $C_{M}, c, \varepsilon_{0}$ to be fixed hereafter, if $0 \leq T<T_{\varepsilon} \leq \tilde{T}_{\varepsilon}$ then

$$
\begin{array}{ll}
A(T)=\left\|\Gamma^{\leq 2} u\right\|_{L_{t}^{q_{*} q_{c}} \mathcal{L}_{r}^{q_{c}} L_{\omega}^{2}(t<T)} & \leq C_{M} \varepsilon^{1 / q_{c}}, \\
B(T)=\left\|\partial \Gamma^{\leq 2} u(T, \cdot)\right\|_{L_{x}^{2}} & \leq C_{M} \varepsilon,
\end{array}
$$

where $q_{*}=\left(q_{c}-1\right) / 2$ as in Lemma 3.3. By Claim 3.7, we fix $C_{M}$ such that $B(0) \leq C_{M} \varepsilon / 4$. We need to show that equation (3.11) implies $A(T) \leq C_{M} \varepsilon^{1 / q_{c}} / 2$ and $B(T) \leq C_{M} \varepsilon / 2$ for above $T$ and $T_{\varepsilon}$.

Part 1: Estimate of $A(t)$.

By Lemma 3.3, and $T<\tilde{T}_{\varepsilon}$, we get

$$
\begin{aligned}
A(T) \leq & c C_{M} \varepsilon / 4^{1 / q_{c}}+c C \varepsilon^{1 / q_{c}-1}\left\|\Gamma^{\leq 2}|u|^{q_{c}}\right\|_{L_{t}^{q_{*}} \mathcal{L}_{r}^{1} L_{\omega}^{2}(t<T)} \\
& +c C \varepsilon^{1 / q_{c}-1}\left\|\Gamma^{\leq 2}|\partial u|^{p}\right\|_{L_{t}^{q_{*}} \mathcal{L}_{r}^{1} L_{\omega}^{2}(t<T)} .
\end{aligned}
$$

For the second term, similar to $n=3$, we have

$$
\begin{aligned}
\left\|\Gamma^{\leq 2}|u|^{q_{c}}\right\|_{L_{t}^{q_{*}} \mathcal{L}_{r}^{1} L_{\omega}^{2}} & \leq C\left\|\left|\Gamma^{\leq 2} u\left\|\left.u\right|^{q_{c}-1}\right\|_{L_{t}^{q_{*}} \mathcal{L}_{r}^{1} L_{\omega}^{2}}+C\left\|\left|\Gamma^{\leq 1} u\right|^{2}|u|^{q_{c}-2}\right\|_{L_{t}^{q_{*}} \mathcal{L}_{r}^{1} L_{\omega}^{2}}\right.\right. \\
& \leq C\left\|\Gamma^{\leq 2} u\right\|_{L_{t}^{q_{*} q_{c}} \mathcal{L}_{r}^{q_{c}} L_{\omega}^{2}}^{q^{2}} \\
& \leq C C_{M}^{q_{c}} \varepsilon
\end{aligned}
$$

and

$$
\begin{aligned}
\left\|\Gamma^{\leq 2}|\partial u|^{p}\right\|_{\mathcal{L}_{r}^{1} L_{\omega}^{2}} & \leq C\left(\left\|\Gamma^{\leq 2} \partial u\right\|_{\mathcal{L}_{r}^{2} L_{\omega}^{2}}\|\partial u\|_{\mathcal{L}_{r}^{2} L_{\omega}^{\infty}}+\left\|\Gamma^{\leq 1} \partial u\right\|_{\mathcal{L}_{r}^{2} L_{\omega}^{4}}^{2}\right)\|\partial u\|_{\mathcal{L}_{r}^{\infty} L_{\omega}^{\infty}}^{p-2} \\
& \leq C\left\|\Gamma^{\leq 2} \partial u\right\|_{L_{x}^{2}}^{2}\|\partial u\|_{L_{x}^{\infty}}^{p-2} \\
& \leq C C_{M}^{p} \varepsilon^{p}\langle t\rangle^{1-p / 2} .
\end{aligned}
$$

Since $p \geq q_{c},\left(1-q_{c} / 2\right) q_{*}=-1, T<\tilde{T}_{\varepsilon}$, we get

$$
\begin{aligned}
A(T) & \leq c C_{M} \varepsilon^{1 / q_{c}}+c C C_{M}^{q_{c}} \varepsilon^{1 / q_{c}-1+1}+c C C_{M}^{p} \varepsilon^{1 / q_{c}-1+p}\left(\int_{0}^{T}\langle t\rangle^{(1-p / 2) q_{*}} \mathrm{~d} t\right)^{1 / q_{*}} \\
& \leq c C_{M} \varepsilon^{1 / q_{c}}+c C C_{M}^{q_{c}} \varepsilon^{1 / q_{c}-1+1}+c C C_{M^{p}}^{p} \varepsilon^{1 / q_{c}-1+p}(\ln (T+2))^{1 / q_{*}} \\
& \leq C_{M} \varepsilon^{1 / q_{c}}\left(c+c C C_{M}^{q_{c}-1}\right) .
\end{aligned}
$$


Part 2: Estimate of $B(t)$.

In this part, by applying the energy inequality, we obtain

$$
B(T) \leq C_{M} \varepsilon / 4+C\left\|\Gamma^{\leq 2}|u|^{q_{c}}\right\|_{L_{t}^{1} L_{x}^{2}(t<T)}+C\left\|\Gamma^{\leq 2}|\partial u|^{p}\right\|_{L_{t}^{1} L_{x}^{2}(t<T)} .
$$

For the second term, by Hölder's inequality, Lemma 3.5 and Lemma 3.6, we have

$$
\begin{aligned}
\left\|\Gamma^{\leq 2}|u|^{q_{c}}\right\|_{L_{t}^{1} L_{x}^{2}} & =\left\|\Gamma^{\leq 2}|u|^{q_{c}}\right\|_{L_{t}^{1} \mathcal{L}_{r}^{2} L_{\omega}^{2}} \\
& \leq C\|\| u\left\|_{\mathcal{L}_{r}^{\infty} L_{\omega}^{\infty}}^{q_{c}-2}\left(\left\|\Gamma^{\leq 2} u\right\|_{\mathcal{L}_{r}^{q_{c}} L_{\omega}^{2}}\|u\|_{\mathcal{L}_{r}^{q_{c}+1} L_{\omega}^{\infty}}+\left\|\Gamma^{\leq 1} u\right\|_{\mathcal{L}_{r}^{4} L_{\omega}^{4}}^{2}\right)\right\|_{L_{t}^{1}} \\
& \leq C\|\| \Gamma^{\leq 1} u\left\|_{L_{x}^{q_{c}}}^{q_{c}-2}\right\| \Gamma^{\leq 2} u\left\|_{\mathcal{L}_{r}^{q_{c}} L_{\omega}^{2}}^{2}\langle t\rangle^{\left(-1 / q_{c}\right)\left(q_{c}-2\right)}\right\|_{L_{t}^{1}} \\
& \leq C\|\| \Gamma^{\leq 2} u\left\|_{\mathcal{L}_{r}^{q_{c}} L_{\omega}^{2}}^{q_{\omega}}\langle t\rangle^{q_{c}-4}\right\|_{L_{t}^{1}} \\
& \leq C\left\|\Gamma^{\leq 2} u\right\|_{L_{t}^{q_{c}^{*} q_{c}} \mathcal{L}_{r}^{q_{c}} L_{\omega}^{2}}^{q_{\omega}}\left\|\langle t\rangle^{q_{c}-4}\right\|_{L_{t}^{1+q_{c}}} \\
& \leq C C_{M}^{q_{c}} \varepsilon^{q_{c}},
\end{aligned}
$$

and the same for the last term

$$
\begin{aligned}
\left\|\Gamma^{\leq 2}|\partial u|^{p}\right\|_{L_{x}^{2}} & \leq C\left\|\Gamma^{\leq 2} \partial u\right\|_{L_{x}^{2}}\|\partial u\|_{L_{x}^{\infty}}^{p-1}+C\left\|\Gamma^{\leq 1} \partial u\right\|_{L_{x}^{4}}^{2}\|\partial u\|_{L_{x}^{\infty}}^{p-2} \\
& \leq C\left\|\Gamma^{\leq 2} \partial u\right\|_{L_{x}^{2}}^{p}\left(\langle t\rangle^{-(1 / 2)(p-1)}+\langle t\rangle^{-(1 / 2-1 / 4) 2-(1 / 2)(p-2)}\right) \\
& \leq C C_{M}^{p} \varepsilon^{p}\langle t\rangle^{(1-p) / 2} .
\end{aligned}
$$

By the definition of $\tilde{T}$ and $\varepsilon_{0}$, we know

$$
B(T) \leq C_{M} \varepsilon\left(1 / 4+C C_{M}^{q_{c}} \varepsilon^{q_{c}-1}+C C_{M}^{p-1} \varepsilon^{p-1}\right) \leq C_{M} \varepsilon\left(1 / 4+C C_{M}^{q_{c}} \varepsilon_{0}^{q_{c}-1}\right) .
$$

Part 3: The boundness of $A(t), B(t)$.

Now, by choosing $\varepsilon_{0}$ and the constant $c$ (in $\tilde{T}_{\varepsilon}$ ) small enough, we conclude $A(T) \leq C_{M} \varepsilon^{1 / q_{c}} / 2$ and $B(T) \leq C_{M} \varepsilon / 2$, which completes the proof.

\subsection{Proof of Claim 3.7.}

Before the discussion, set $h=\left\{\partial_{\bar{x}}^{\leq 1} f, g\right\}$, by equation (1.1) we see

$$
\begin{aligned}
\left|\left\{\partial_{x}^{\leq 3} u, \partial_{x}^{\leq 2} \partial_{t} u\right\}\right|_{t=0} & \leq C \varepsilon\left|\partial_{x}^{\leq 2} h\right| \\
\left|\partial_{x}^{\leq 1} \partial_{t}^{2} u\right|_{t=0} & =\left|\partial_{x}^{\leq 1}\left(|\partial u|^{p}+|u|^{q_{c}}+\Delta u\right)\right|_{t=0} \\
& \leq C \varepsilon\left|\left\{\partial_{x}^{\leq 1} h|h|^{p-1},|h|^{q_{c}}, \partial_{x}^{\leq 2} h\right\}\right| \\
\left|\partial_{t}^{3} u\right|_{t=0} & \leq C\left|\left\{\partial \partial_{t} u|\partial u|^{p-1}, \partial_{t} u|u|^{q_{c}-1}, \partial_{t} \Delta u\right\}\right|_{t=0} \\
& \leq C\left|\left\{\left(|\partial u|^{p}+|u|^{q_{c}}+\Delta u\right)|\partial u|^{p-1}, \partial_{x} \partial_{t} u|\partial u|^{p-1}, \partial_{t} u|u|^{q_{c}-1}, \partial_{t} \Delta u\right\}\right| \\
& \leq C \varepsilon\left|\left\{|h|^{2 p-1},|h|^{q_{c}+p-1}, \partial_{x}^{\leq 1} h|h|^{p-1},|h|^{q_{c}}, \partial_{x}^{\leq 2} h\right\}\right| .
\end{aligned}
$$

Set $M=\Lambda+\Lambda^{p}+\Lambda^{q_{c}}+\Lambda^{2 p-1}+\Lambda^{q_{c}+p-1}$, we want to show that all of the initial norms can be controlled by $C \varepsilon M$, where $C$ does not depend on $\varepsilon$ and $M$. 
We begin with $A(0)=\left\|\Gamma^{\leq 2} u(0, \cdot)\right\|_{\mathcal{L}_{r}^{q c} L_{\omega}^{3}}(n=3)$, here we get

$$
\begin{aligned}
\left\|\Gamma^{\leq 2} u(0, \cdot)\right\|_{\mathcal{L}_{r}^{q_{c}} L_{\omega}^{3}} & \leq C\left\|\Gamma^{\leq 3} u(0, \cdot)\right\|_{L_{x}^{q_{c}}} \\
& \leq C\left\|\langle x\rangle^{5} \partial^{\leq 3} u(0, \cdot)\right\|_{L_{x}^{\infty}}\left\|\langle x\rangle^{-2}\right\|_{L_{x}^{q_{c}}} \\
& \leq C \varepsilon M .
\end{aligned}
$$

Similarly, for $B(0)=\left\|\partial \Gamma^{\leq 2} u(0, \cdot)\right\|_{L_{x}^{2}}(n=2,3)$, we have

$$
\left\|\partial \Gamma^{\leq 2} u(0, \cdot)\right\|_{L_{x}^{2}} \leq C\left\|\langle x\rangle^{5} \partial^{\leq 3} u(0, \cdot)\right\|_{L_{x}^{\infty}}\left\|\langle x\rangle^{-3}\right\|_{L_{x}^{2}} \leq C \varepsilon M .
$$

For $\left\|r^{1-2 / q_{c}} \partial_{\omega}^{\leq 1} \Gamma^{\leq 2} u(0, \cdot)\right\|_{\mathcal{L}_{r}^{q_{c}} L_{\omega}^{3 / 2}}(n=3)$ which comes from right hand side (RHS) of equation (3.1), we see

$$
\begin{aligned}
\left\|r^{1-2 / q_{c}} \partial_{\omega}^{\leq 1} \Gamma^{\leq 2} u(0, \cdot)\right\|_{\mathcal{L}_{r}^{q_{c}} L_{\omega}^{3 / 2}} & \leq C\left\|\langle x\rangle^{4-2 / q_{c}} \partial^{\leq 3} f\right\|_{L_{x}^{q_{c}}} \\
& \leq C\left\|\langle x\rangle^{5} \partial^{\leq 3} u(0, \cdot)\right\|_{L_{x}^{\infty}}\left\|\langle x\rangle^{-1-2 / q_{c}}\right\|_{L_{x}^{q_{c}}} \\
& \leq C \varepsilon M .
\end{aligned}
$$

The other three terms from equation (3.1) can be controlled by similar arguments. At last, for the term $\left\|\Gamma^{\leq 2}\left(u, \partial_{t} u\right)(0, \cdot)\right\|_{X^{b}}(n=2)$ which comes from equation (3.2), we have

$$
\left\|\Gamma^{\leq 2}\left(u, \partial_{t} u\right)(0, \cdot)\right\|_{X^{b}} \leq\left\|\Gamma^{\leq 2}\left(u, \partial_{t} u\right)(0, \cdot)\right\|_{\left(H^{1}, L^{2}\right)} .
$$

Through the previous discussion, we finish the proof of the Claim 3.7.

\section{Proof of Theorem 1.3}

Before the proof, we consider a simple coordinate transform with $\left.\left(u, u_{t}\right)\right|_{t=2}=$ $(f, g),\left.\left(v, v_{t}\right)\right|_{t=2}=(\tilde{f}, \tilde{g})$. Due to the property of symmetry, $(u, v)$ solve the equivalent 1-D integral equations in $t \geq 2$

$$
\begin{aligned}
& u(t, r)=\varepsilon u_{o}(t, r)+L|v|^{q}(t, r), \\
& v(t, r)=\varepsilon v_{o}(t, r)+L\left|\partial_{t} u\right|^{p}(t, r),
\end{aligned}
$$

where

$$
\begin{aligned}
u_{o}(t+2, r) & =\frac{1}{2 r}\left((r+t) f(r+t)+(r-t) f(r-t)+\int_{r-t}^{r+t} \rho g(\rho) \mathrm{d} \rho\right), \\
v_{o}(t+2, r) & =\frac{1}{2 r}\left((r+t) \tilde{f}(r+t)+(r-t) \tilde{f}(r-t)+\int_{r-t}^{r+t} \rho \tilde{g}(\rho) \mathrm{d} \rho\right), \\
L F(t, r) & =\frac{1}{2 r} \int_{0}^{t} \int_{r-t+s}^{r+t-s} \rho F(s, \rho) \mathrm{d} \rho \mathrm{d} s .
\end{aligned}
$$

for $t \geq 0$. Here for convenience we consider $\left.u\right|_{t<2}=\left.v\right|_{t<2}=0$.

Here we denote $f(|x|)=f(-|x|)=f(x)$ and the rest is similar. Then the lower limits of the integrals (to $\rho$ ) may be replaced by $|r-t|$ or $|r-(t-s)|$ because of the symmetric assumption. To control the iteration procedure, we need to estimate 
some derivatives. With the notation $w=\partial_{t} u$, we find that

$$
\begin{aligned}
w(t, r) & =\varepsilon \partial_{t} u_{o}(t, r)+r^{-1} K_{+}|v|^{q}(t, r), \\
v(t, r) & =\varepsilon v_{o}(t, r)+L|w|^{p}(t, r), \\
\partial_{r}\{r v(t, r)\} & =\varepsilon \partial_{r}\left(r v_{o}(t, r)\right)+K_{-}|w|^{p}(t, r),
\end{aligned}
$$

where

$$
K_{ \pm} F(t, r)=\frac{1}{2} \int_{0}^{t}(r+t-s) F(s, r+t-s) \pm(r-t+s) F(s, r-t+s) \mathrm{d} s .
$$

To control the norm of $(w, v)$, we set

$$
\|(w, v)\|=\left\|\omega_{1} w\right\|_{L_{t, r}^{\infty}}+\left\|\omega_{2} v\right\|_{L_{t, r}^{\infty}}+\left\|\omega_{3} r \partial_{r} v\right\|_{L_{t, r}^{\infty}}
$$

where weight functions $\omega_{1}, \omega_{2}, \omega_{3}$ are defined by

$$
\begin{aligned}
& \omega_{1}(t, r)= \begin{cases}\langle r\rangle\langle t-r\rangle^{\mu / p+q-2} & (r<t / 2), \\
\langle t-r\rangle^{\mu / p}\langle t+r\rangle^{q-1} & (r \geq t / 2) ;\end{cases} \\
& \omega_{2}(t, r)= \begin{cases}\langle r\rangle^{p-2}\langle t+r\rangle^{3-p+\mu / p q} & (r<t / 2), \\
\langle t-r\rangle^{\mu / p q}\langle t+r\rangle & (r \geq t / 2) ;\end{cases} \\
& \omega_{3}(t, r)=\langle t-r\rangle^{\mu+p q-2 p}
\end{aligned}
$$

for $t \geq 0$ and $r \geq 0$, with a fixed $\mu<1$ which satisfies

$$
-\mu-p q+2 p \leq p-3-\mu / p q
$$

Now we consider the system of integral equations (4.1)-(4.3) in the close subset of complete metric space

$$
\begin{gathered}
X_{\varepsilon}=\left\{(w, v): w, v, r \partial_{r} v \in C([2, \infty) \times \mathbb{R}),\|(w, v)\| \leq C_{1} \varepsilon\right. \\
\operatorname{supp}(w, v) \subset\{t-r \geq 1, t \geq 2\}\}
\end{gathered}
$$

where $C_{1}$ will be determined later.

Lemma 4.1. Suppose that (1.9) is satisfied, $(w, v) \in X_{\varepsilon}$. Then we have

$$
\begin{aligned}
& \left\|\omega_{2} L|w|^{p}\right\|_{L_{t, r}^{\infty}} \leq C\left\|\omega_{1} w\right\|_{L_{t, r}^{\infty}}^{p}, \\
& \left\|\omega_{1} r^{-1} K_{+}|v|^{q}\right\|_{L_{t, r}^{\infty}} \leq C\left\|\omega_{2} v\right\|_{L_{t, r}^{\infty}}^{q}+C\left\|\omega_{2} v\right\|_{L_{t, r}^{\infty}}^{q-1}\left\|\omega_{3} r \partial_{r} v\right\|_{L_{t, r}^{\infty},}, \\
& \left\|\omega_{3} K_{-}|w|^{p}\right\|_{L_{t, r}^{\infty}}^{\infty} \leq C\left\|\omega_{1} w\right\|_{L_{t, r}^{\infty}}^{p} .
\end{aligned}
$$

Lemma 4.2. Suppose that (1.9) is satisfied, $(w, v),(\bar{w}, \bar{v}) \in X_{\varepsilon}$. Set

$$
\begin{aligned}
& \tilde{\omega}_{1}= \begin{cases}r\langle t-r\rangle^{\mu / p+q-2} & (r<t / 2), \\
\langle t-r\rangle^{\mu / p}\langle t+r\rangle^{q-1} & (r \geq t / 2) ;\end{cases} \\
& \tilde{\omega}_{2}= \begin{cases}r^{p-2}\langle t+r\rangle^{3-p+\mu / p q} & (r<t / 2), \\
\langle t-r\rangle^{\mu / p q}\langle t+r\rangle & (r \geq t / 2) .\end{cases}
\end{aligned}
$$

Then we have

$$
\begin{aligned}
& \left\|\tilde{\omega}_{2} L\left(|w|^{p}-|\bar{w}|^{p}\right)\right\|_{L_{t, r}^{\infty}} \leq C\left\|\tilde{\omega}_{1}(w, \bar{w})\right\|_{L_{t, r}^{\infty}}^{p-1}\left\|\tilde{\omega}_{1}(w-\bar{w})\right\|_{L_{t, r}^{\infty},} \\
& \left\|\tilde{\omega}_{1} r^{-1} K_{+}\left(|v|^{q}-|\bar{v}|^{q}\right)\right\|_{L_{t, r}^{\infty}} \leq C\left\|\tilde{\omega}_{2}(v, \bar{v})\right\|_{L_{t, r}^{\infty}}^{q-1}\left\|\tilde{\omega}_{2}(v-\bar{v})\right\|_{L_{t, r}^{\infty}} .
\end{aligned}
$$


Here, we apply the fixed point theorem with mapping

$$
P:(w, v) \mapsto(P w, P v):=\left(\varepsilon \partial_{t} u_{o}+r^{-1} K_{+}|v|^{q}, \varepsilon v_{o}+L|w|^{p}\right) .
$$

Firstly we check that $P$ is well defined in $X_{\varepsilon} \rightarrow X_{\varepsilon}$. By expression (4.1)-(4.3), it is obvious that $\operatorname{supp}(P w, P v) \subset\{t-r \geq 1, t \geq 2\}$ and $P w, P v, r \partial_{r} P v \in C\left(\mathbb{R}^{+} \times \mathbb{R}\right)$. To estimate $\|(P w, P v)\|$, we begin with

$$
\left\|\omega_{1} P w\right\|_{L_{t, r}^{\infty}} \leq \varepsilon\left\|\omega_{1} \partial_{t} u_{o}\right\|_{L_{t, r}^{\infty}}+\left\|\omega_{1} r^{-1} K_{+}|v|^{q}\right\|_{L_{t, r}^{\infty}} .
$$

Since $f \in C^{2}, g \in C^{1}$, and $\operatorname{supp} u_{o} \subset\{(t, r): 3 \geq t-r \geq 1\}$ where $\omega_{1} \lesssim\langle t\rangle$, we see

$$
\left\|\omega_{1} \partial_{t} u_{o}\right\|_{L_{t, r}^{\infty}} \leq C_{f, g}
$$

Moreover, by (4.6), and $(w, v) \in X_{\varepsilon}$, we conclude

$$
\left\|\omega_{1} P w\right\|_{L_{t, r}^{\infty}} \leq C_{f, g} \varepsilon+C \varepsilon^{q} .
$$

The estimates for the remaining terms are similar, noticing $r \partial_{r} P v=\partial_{r}(r P v)-$ $P v$ and $\omega_{3} \lesssim \omega_{2}$, by (4.5) and (4.7) we finally have

$$
\|(w, v)\| \leq C_{f, g, \tilde{f}, \tilde{g}} \varepsilon+C \varepsilon^{p}+C \varepsilon^{q} \leq C_{1} \varepsilon
$$

for $C_{1} \geq 2 C_{f, g, \tilde{f}, \tilde{g}}$ and $\varepsilon$ small enough.

Similarly, by (4.8) and (4.9) we have $P$ is contraction in a weaker sense. However it is enough to obtain the fixed point $(u, v)$ which solves (4.1)-(4.3). So we complete the proof.

\subsection{Proof of (4.5) and (4.8).}

First we prove (4.5). Let $r>0$. Considering $D=\{(s, \rho): t-r \leq s+\rho \leq t+r, 1 \leq$ $s-\rho \leq t-r\}$ is the influence domain of $(t, r)$ intersect with $\{(s, \rho): s-\rho \geq 1\}$. Set $D_{1}=D \cap\{(s, \rho): \rho<s / 2\}, D_{2}=D \cap\{(s, \rho): \rho \geq s / 2\}$, then we find

$$
\begin{aligned}
\left.\left|\omega_{2} L\right| w\right|^{p} \mid & \leq \omega_{2} \frac{C}{r} \int_{D} \rho \omega_{1}^{-p}(s, \rho)\left\|\omega_{1} w\right\|_{L_{t, r}^{\infty}}^{p} \mathrm{~d} \rho \mathrm{d} s \\
& =C \omega_{2}\left\|\omega_{1} w\right\|_{L_{t, r}^{\infty}}^{p} \frac{1}{r}\left(\int_{D_{1}}+\int_{D_{2}}\right) \rho \omega_{1}^{-p}(s, \rho) \mathrm{d} \rho \mathrm{d} s .
\end{aligned}
$$

Part 1: $(s, \rho) \in D_{1}$.

Here $\langle s\rangle \approx\langle s-\rho\rangle \approx\langle s+\rho\rangle$, take $\tau=s+\rho, \sigma=s-\rho$, by (4.4) we obtain

$$
\begin{aligned}
\frac{1}{r} \int_{D_{1}} \rho \omega_{1}^{-p}(s, \rho) \mathrm{d} \rho \mathrm{d} s & =\frac{1}{r} \int_{D_{1}} \rho\langle\rho\rangle^{-p}\langle s-\rho\rangle^{-\mu-p q+2 p} \mathrm{~d} \rho \mathrm{d} s \\
& \leq \frac{C}{r} \int_{t-r}^{t+r} \int_{(t-r) / 3}^{t-r}\langle\tau-\sigma\rangle^{1-p}\langle\tau\rangle^{-\mu-p q+2 p} \mathrm{~d} \sigma \mathrm{d} \tau \\
& \leq \frac{C}{r} \int_{t-r}^{t+r}\langle\tau-t+r\rangle^{2-p}\langle\tau\rangle^{p-3-\mu / p q} \mathrm{~d} \tau .
\end{aligned}
$$

Part 1.1: $r<t / 2$.

Here $t-r \approx t \approx t+r$, we conclude

$$
\begin{aligned}
R H S \text { of }(4.11) & \leq C\langle t+r\rangle^{p-3-\mu / p q} r^{-1}\left((r+1)^{3-p}-1^{3-p}\right) \\
& \leq C\langle t+r\rangle^{p-3-\mu / p q}\langle r\rangle^{2-p} \\
& =C \omega_{2}^{-1} .
\end{aligned}
$$

Part 1.2: $r \geq t / 2$. 
Here $r \approx t \approx t+r$, similarly we have

$$
\begin{aligned}
& R H S \text { of }(4.11) \leq C\langle t\rangle^{-1}\left(\langle t-r\rangle^{p-3-\mu / p q} \int_{t-r}^{2(t-r)}\langle\tau-t+r\rangle^{2-p} \mathrm{~d} \tau\right. \\
&\left.\quad+\int_{2(t-r)}^{t+r}\langle\tau\rangle^{-1-\mu / p q} \mathrm{~d} \tau\right) \\
& \leq C\langle t\rangle^{-1}\langle t-r\rangle^{-\mu / p q} \\
& \leq C \omega_{2}^{-1} .
\end{aligned}
$$

Part 2: $(s, \rho) \in D_{2}$.

Here $\langle\rho\rangle \approx\langle s\rangle \approx\langle s+\rho\rangle$, take $\tau=s+\rho, \sigma=s-\rho$, then we conclude

$$
\begin{aligned}
\frac{1}{r} \int_{D_{2}} \rho \omega_{1}^{-p}(s, \rho) \mathrm{d} \rho \mathrm{d} s & =\frac{1}{r} \int_{D_{2}} \rho\langle s-\rho\rangle^{-\mu}\langle s+\rho\rangle^{-p(q-1)} \mathrm{d} \rho \mathrm{d} s \\
& \leq \frac{C}{r} \int_{t-r}^{t+r}\langle\tau\rangle^{1-p(q-1)} \mathrm{d} \tau \int_{1}^{t-r}\langle\sigma\rangle^{-\mu} \mathrm{d} \sigma .
\end{aligned}
$$

Part 2.1: $r<t / 2$.

Here we have

$$
R H S \text { of }(4.14) \leq C\langle t-r\rangle^{1-p(q-1)}\langle t-r\rangle^{1-\mu} \leq C \omega_{2}^{-1} .
$$

Part 2.2: $r \geq t / 2$.

Here we have

$$
R H S \text { of }(4.14) \leq C\langle t\rangle^{-1}\langle t-r\rangle^{2-p(q-1)}\langle t-r\rangle^{1-\mu} \leq C \omega_{2}^{-1} .
$$

Thus (4.10)-(4.16) give $\left.\left|\omega_{2} L\right| w\right|^{p} \mid \leq C\left\|\omega_{1} w\right\|_{L_{t, r}^{\infty}}^{p}$ which completes the proof of (4.5). The proof of (4.8) is similar, since that $\left.|| a\right|^{q}-|b|^{q}\left|\lesssim\left(|a|^{q-1}+|b|^{q-1}\right)\right| a-b \mid$ for $q>1$.

\subsection{Proof of (4.6) and (4.9).}

For (4.6), we divide the proof into two main cases: $r \geq 1 / 4$ and $r<1 / 4$.

Part 1: $r \geq 1 / 4$.

For this situation, we see $r \approx\langle r\rangle$. Similar to the proof of (4.5) we have (4.17)

$$
\begin{aligned}
\left.\left|\omega_{1} r^{-1} K_{+}\right| v\right|^{q} \mid & \leq\left\|\omega_{2} v\right\|_{L_{t, r}^{\infty}}^{q} \omega_{1} r^{-1} \int_{0}^{t} \frac{(r+t-s)}{\omega_{2}(s, r+t-s)^{q}}+\frac{|r-t+s|}{\omega_{2}(s,|r-t+s|)^{q}} \mathrm{~d} s \\
& \equiv\left\|\omega_{2} v\right\|_{L_{t, r}^{\infty}}^{q} \omega_{1} r^{-1} \int_{0}^{t} I+I I \mathrm{~d} s
\end{aligned}
$$

Part 1.1: $r<t / 2$. Estimates about $I$.

In this part, we obtain $\omega_{1} r^{-1} \approx\langle t-r\rangle^{\mu / p+q-2}$.

Part 1.1.1: $0 \leq s<2(r+t) / 3$. 
This means $r+t-s>s / 2$, then we conclude

$$
\begin{aligned}
\int_{0}^{2(t+r) / 3} I \mathrm{~d} s & =\int_{0}^{2(t+r) / 3}(r+t-s)\langle 2 s-r-t\rangle^{-\mu / p}\langle r+t\rangle^{-q} \mathrm{~d} s \\
& \leq C\langle t+r\rangle^{1-q} \int_{0}^{2(t+r) / 3}\langle 2 s-r-t\rangle^{-\mu / p} \mathrm{~d} s \\
& \leq C\langle t-r\rangle^{2-q-\mu / p} \\
& \leq C r \omega_{1}^{-1} .
\end{aligned}
$$

Part 1.1.2: $2(r+t) / 3 \leq s \leq t$.

This means $r+t-s \leq s / 2$, then similarly we have

$$
\begin{aligned}
\int_{2(t+r) / 3}^{t} I \mathrm{~d} s & =\int_{2(t+r) / 3}^{t}(r+t-s)\langle r+t-s\rangle^{-q(p-2)}\langle r+t\rangle^{-3 q+p q-\mu / p} \mathrm{~d} s \\
& \leq C\langle t+r\rangle^{2-q(p-2)}\langle r+t\rangle^{-3 q+p q-\mu / p} \\
& \leq C r \omega_{1}^{-1}
\end{aligned}
$$

Part 1.2: $r \geq t / 2$. Estimates about $I$.

Here we always have $r+t-s \geq s / 2$. Then it is similar to (4.18).

Part 1.3: $r \geq t / 2$. Estimates about $I I$.

Part 1.3.1: $0 \leq s<t-r$.

Here $|r-t+s|=t-s-r$. It is similar to (4.18)-(4.19).

Part 1.3.2: $t-r \leq s<2(t-r)$.

Here $r-t+s<s / 2$, then we have

$$
\begin{aligned}
\int_{t-r}^{2(t-r)} I I \mathrm{~d} s & =\int_{t-r}^{2(t-r)}(r-t+s)\langle r-t+s\rangle^{-q(p-2)}\langle r-t+2 s\rangle^{-3 q+p q-\mu / p} \mathrm{~d} s \\
& \leq C\langle t-r\rangle^{2-q(p-2)}\langle t-r\rangle^{-3 q+p q-\mu / p} \\
& \leq C r \omega_{1}^{-1} .
\end{aligned}
$$

Part 1.3.3: $2(t-r) \leq s<t$.

Here $r-t+s \geq s / 2$, then we also have

$$
\begin{aligned}
\int_{2(t-r)}^{t} I I \mathrm{~d} s & =\int_{2(t-r)}^{t}(r-t+s)\langle t-r\rangle^{-\mu / p}\langle r-t+2 s\rangle^{-q} \mathrm{~d} s \\
& \leq C\langle t-r\rangle^{-\mu / p} \int_{2(t-r)}^{t}\langle r-t+2 s\rangle^{1-q} \mathrm{~d} s \\
& \leq C\langle t-r\rangle^{-\mu / p}\langle t+r\rangle^{2-q} \\
& \leq C r \omega_{1}^{-1} .
\end{aligned}
$$

Part 1.4: $r<t / 2$. Estimates about $I I$.

Part 1.4.1: $0 \leq s<t-r$.

It is similar to (4.18)-(4.19).

Part 1.4.2: $t-r \leq s \leq t$. 
Here we have

$$
\begin{aligned}
\int_{t-r}^{t} I I \mathrm{~d} s & =\int_{t-r}^{t}(r-t+s)\langle r-t+s\rangle^{-q(p-2)}\langle r-t+2 s\rangle^{-3 q+p q-\mu / p} \mathrm{~d} s \\
& \leq C\langle r\rangle^{2-q(p-2)}\langle t+r\rangle^{-3 q+p q-\mu / p} \\
& \leq C r \omega_{1}^{-1} .
\end{aligned}
$$

Part 2: $r<1 / 4$.

In this part $\langle r\rangle \approx 1$. For the convenience of proof, we set $r^{+}=r+t-s$, $r^{-}=r-t+s$. Then we get

$$
\left.\left|\omega_{1} r^{-1} K_{+}\right| v\right|^{q}\left|\leq C \omega_{1} r^{-1} \int_{0}^{t}\right| r^{+}|v|^{q}\left(s, r^{+}\right)+r^{-}|v|^{q}\left(s,\left|r^{-}\right|\right) \mid \mathrm{d} s
$$

where

$$
\begin{aligned}
\left.\left|r^{+}\right| v\right|^{q}\left(s, r^{+}\right)+r^{-}|v|^{q}\left(s,\left|r^{-}\right|\right) \mid \leq & \left.\left|r^{+}\right| v\right|^{q}\left(s, r^{+}\right)+r^{-}|v|^{q}\left(s, r^{+}\right) \mid \\
& +\left.\left|r^{-}\right| v\right|^{q}\left(s, r^{+}\right)-r^{-}|v|^{q}\left(s,\left|r^{-}\right|\right) \mid
\end{aligned}
$$

then

$$
\begin{aligned}
R H S \text { of }(4.23) & \leq C \omega_{1}\left(\int_{0}^{t}|v|^{q}\left(s, r^{+}\right) \mathrm{d} s+\left.r^{-1} \int_{0}^{t}\left|r^{-}\right||| v\right|^{q}\left(s, r^{+}\right)-|v|^{q}\left(s,\left|r^{-}\right|\right) \mid \mathrm{d} s\right) \\
& \equiv C \omega_{1} I+C \omega_{1} I I .
\end{aligned}
$$

Part 2.1: Estimates about $I$.

It is similar to (4.18)-(4.19).

Part 2.2: Estimates about $I I$.

For this part, we should consider $\partial_{r} v$. Since $r^{+}-\left|r^{-}\right| \leq 2 r$, we obtain

$$
\left.r^{-1}|| v\right|^{q}\left(s, r^{+}\right)-\left.|v|^{q}\left(s,\left|r^{-}\right|\right)|\leq C| \partial_{r} v\left(s, r^{*}\right)|| v\right|^{q-1}\left(s, r^{*}\right)
$$

for $\left|r^{-}\right|<r^{*}(s)<r^{+}$. Then we have

$$
\begin{aligned}
I I & \leq C \int_{0}^{t}\left|r^{-}\left\|\partial_{r} v\left(s, r^{*}\right)\right\| v\right|^{q-1}\left(s, r^{*}\right) \mathrm{d} s \\
& \leq C\left\|\omega_{2} v\right\|_{L_{t, r}^{\infty}}^{q-1}\left\|\omega_{3} r \partial_{r} v\right\|_{L_{t, r}^{\infty}} \int_{0}^{t}\left(\omega_{2}^{-(q-1)} \omega_{3}^{-1}\right)\left(s, r^{*}\right) \mathrm{d} s \\
& \equiv C\left\|\omega_{2} v\right\|_{L_{t, r}^{\infty}}^{q-1}\left\|\omega_{3} r \partial_{r} v\right\|_{L_{t, r}^{\infty}} \int_{0}^{t} I I I \mathrm{~d} s
\end{aligned}
$$

Here we notice $\langle t\rangle \lesssim\left\langle\left|r^{-}\right|+s+r\right\rangle \lesssim\left\langle r^{*}+s\right\rangle$.

Part 2.2.1: $r^{*}<s / 2$. 
Here $\left\langle s \pm r^{*}\right\rangle \gtrsim\left\langle r^{*}\right\rangle \gtrsim\langle t-s\rangle$, by (4.4), we see

$$
\begin{aligned}
\omega_{1} \int_{2 r^{*}<s<t} I I I \mathrm{~d} s & \leq C \int_{2 r^{*}<s<t}\left\langle r^{*}\right\rangle^{(1-q)(p-2)} \\
& \times\left\langle s-r^{*}\right\rangle^{(1 / p-1)(\mu+p q-2 p)+(1-q)(3-p+\mu / p q)} \mathrm{d} s \\
& \leq C \int_{2 r^{*}<s<t}\left\langle r^{*}\right\rangle^{-1-\mu-p q+2 p+\mu / p q} \mathrm{~d} s \\
& \leq C \int_{2 r^{*}<s<t}\langle t-s\rangle^{p-4} \mathrm{~d} s \\
& \leq C .
\end{aligned}
$$

Part 2.2.2: $r^{*} \geq s / 2$.

Here $\left\langle s+r^{*}\right\rangle \gtrsim\left\langle s-r^{*}\right\rangle \gtrsim\langle 2 s-t\rangle$, similarly we have

$$
\omega_{1} \int_{s<2 r^{*} \wedge t} I I I \mathrm{~d} s \leq C \int_{s<2 r^{*} \wedge t}\langle 2 s-t\rangle^{p-4} \mathrm{~d} s \leq C .
$$

In summary, we complete the proof. To verify (4.9), without distinguishing whether $r>1 / 4$ or not we have a proof just like (4.10)-(4.22).

\subsection{Proof of (4.7).}

We follow the same process as before

$$
\left.\left|\omega_{3} K_{-}\right| w\right|^{p} \mid \leq\left\|\omega_{1} v\right\|_{L_{t, r}^{\infty}}^{p} \omega_{3} \int_{0}^{t} \frac{r+t-s}{\omega_{1}(s, r+t-s)^{p}}+\frac{|r-t+s|}{\omega_{1}(s,|r-t+s|)^{p}} \mathrm{~d} s .
$$

Both part in the integration is similar to the last proof. Here we only show the proof of $r \geq t / 2,(t-r) \leq s$ for $|r-t+s|$ part.

Part 1: $t-r \leq s<2(t-r)$.

Here $r-t+s<s / 2$, then we see

$$
\begin{aligned}
\int_{t-r}^{2(t-r)} \frac{r-t+s}{\omega_{1}(s, r-t+s)^{p}} \mathrm{~d} s & =\int_{t-r}^{2(t-r)}(r-t+s)\langle r-t+s\rangle^{-p}\langle r-t+2 s\rangle^{-\mu-p q+2 p} \mathrm{~d} s \\
& \leq C\langle t-r\rangle^{-\mu-p q+2 p} \\
& \leq C \omega_{3}^{-1} .
\end{aligned}
$$

Part 2: $2(t-r) \leq s \leq t$.

Here $r-t+s \geq s / 2$, then we know

$$
\begin{aligned}
\int_{2(t-r)}^{t} \frac{r-t+s}{\omega_{1}(s, r-t+s)^{p}} \mathrm{~d} s & =\int_{2(t-r)}^{t}(r-t+s)\langle t-r\rangle^{-\mu}\langle r-t+2 s\rangle^{-p q+p} \mathrm{~d} s \\
& \leq C\langle t-r\rangle^{-\mu} \int_{2(t-r)}^{t}\langle r-t+2 s\rangle^{1-p q+p} \mathrm{~d} s \\
& \leq C\langle t-r\rangle^{-\mu}\langle t-r\rangle^{2-p q+p} \\
& \leq C \omega_{3}^{-1}
\end{aligned}
$$

which complete the proof. 


\section{Proof of Theorem 1.4}

Following [18], we introduce two positive test functions

$$
\phi(x)=\int_{S^{n-1}} e^{x \cdot \omega} \mathrm{d} \omega, \quad \psi(t, x)=e^{-t} \phi(x),
$$

where it is understood that $\phi(x)=e^{x}+e^{-x}$ when $n=1$. It is well known that we have

Proposition 5.1. For $\phi, \psi$ defined above, then we have

$$
\begin{aligned}
& \psi(t, x) \leq C\langle r\rangle^{-(n-1) / 2} e^{r-t}, \\
& \|\psi(t, x)\|_{L_{x}^{s}(r<t+1)} \leq C\langle t\rangle^{(n-1)(1 / s-1 / 2)},
\end{aligned}
$$

for any $s \in[1, \infty]$.

We will prove Theorem 1.4 by contradiction. Suppose there are global weak solutions of (1.5) with $\operatorname{supp}(u, v) \subset\{(t, x):|x| \leq t+1\}$ and (1.11). As in [20], we define

$$
F(t)=\int_{\mathbb{R}^{n}} u(t, x) \psi(t, x) \mathrm{d} x, \quad G(t)=\int_{\mathbb{R}^{n}} v(t, x) \psi(t, x) \mathrm{d} x .
$$

Since $u, v, \partial_{t} u, \partial_{t} v \in C\left(\mathbb{R}_{+} ; L^{1}\left(\mathbb{R}^{n}\right)\right)$, we know that $F, G \in C^{1}\left(\mathbb{R}_{+}\right)$and

$$
F^{\prime}(t)=\int_{\mathbb{R}^{n}} u_{t}(t, x) \psi(t, x)+u(t, x) \psi_{t}(t, x) \mathrm{d} x=\int_{\mathbb{R}^{n}} u_{t}(t, x) \psi(t, x) \mathrm{d} x-F(t) .
$$

By the definition of weak solutions to (1.5), with $\psi$ as test function, combined with the assumption $v \in C\left(\mathbb{R}_{+} ; L^{q}\right)$, we know that $F \in C^{2}\left(\mathbb{R}_{+}\right)$and

$$
F^{\prime \prime}(t)=\int_{\mathbb{R}^{n}}|v|^{q}(t, x) \psi(t, x) \mathrm{d} x-2 F^{\prime}(t) .
$$

Similarly, as $\partial_{t} u \in C\left(\mathbb{R}_{+} ; L^{p}\right)$, we conclude $G \in C^{2}\left(\mathbb{R}_{+}\right)$and

$$
G^{\prime \prime}(t)=\int_{\mathbb{R}^{n}}\left|\partial_{t} u(t, x)\right|^{p} \psi(t, x) \mathrm{d} x-2 G^{\prime}(t) .
$$

Since $\operatorname{supp}(u, v) \subset\{(t, x):|x| \leq t+1\}$, using Hölder's inequality and Proposition 5.1, we know that

$$
\begin{gathered}
F^{\prime \prime}(t)+2 F^{\prime}(t)=\int_{\mathbb{R}^{n}}|v(t, x)|^{q} \psi(t, x) \mathrm{d} x \geq C\langle t\rangle^{-\frac{n-1}{2}(q-1)}|G(t)|^{q}, \\
G^{\prime \prime}(t)+2 G^{\prime}(t)=\int_{\mathbb{R}^{n}}\left|\partial_{t} u\right|^{p} \psi \mathrm{d} x \geq C\langle t\rangle^{-\frac{n-1}{2}(p-1)}\left|F^{\prime}(t)+F(t)\right|^{p} .
\end{gathered}
$$

Notice that we have $F^{\prime \prime}(t)+2 F^{\prime}(t) \geq 0$ by (5.1). By the assumption of data we have $F^{\prime}(0)=\int_{\mathbb{R}^{n}}(g-f) \phi \mathrm{d} x \geq 0$, then it is easy to conclude that $F^{\prime}(t) \geq 0$, for any $t \geq 0$. Moreover, since $F(0)=\int_{\mathbb{R}^{n}} f \phi \mathrm{d} x \geq 0$, we have $F(t) \geq 0$ for all $t \geq 0$. Then it is obvious that

$$
\left|F^{\prime}(t)+F(t)\right| \geq \frac{1}{2}\left|F^{\prime}(t)+2 F(t)\right|
$$


By a similar argument, since $\tilde{f}$ does not vanish identically, we have $G^{\prime}(t) \geq 0$, $G(t) \geq C \varepsilon$. Set $H(t)=F^{\prime}(t)+2 F(t)$, then we have $H \in C^{1}\left(\mathbb{R}_{+}\right), G \in C^{2}\left(\mathbb{R}_{+}\right)$and

$$
\left\{\begin{array}{l}
H^{\prime}(t) \geq C\langle t\rangle^{-\frac{n-1}{2}(q-1)} G(t)^{q} \\
H(t) \geq 0, \quad H^{\prime}(t) \geq 0 \\
G^{\prime \prime}(t)+2 G^{\prime}(t) \geq C\langle t\rangle^{-\frac{n-1}{2}(p-1)} H(t)^{p} \\
G(t) \geq C \varepsilon, \quad G^{\prime}(t) \geq 0
\end{array}\right.
$$

Lemma 5.2. For system (5.2), assume (1.10) is satisfied, then for any $M>0$, there exist constants $A, T$, which may depend on $\varepsilon$, such that we have

$$
G(t) \geq A\langle t\rangle^{M}
$$

for any $t \geq T$.

Lemma 5.3. Under the same assumption of Lemma 5.2, there are no $H \in C^{1}\left(\mathbb{R}_{+}\right)$, $G \in C^{2}\left(\mathbb{R}_{+}\right)$satisfying the system (5.2).

This gives the desired contradiction, which completes the proof.

\subsection{Proof of Lemma 5.2.}

At first, we see that

$$
G(t) \geq A_{0}\langle t\rangle^{\alpha_{0}}, \forall t \geq T_{0}
$$

with $A_{0}=C \varepsilon, \alpha_{0}=0, T_{0}=1$. We claim that for any $m \geq 0$, there exists $A_{m}>0$ such that we have

$$
G(t) \geq A_{m}\langle t\rangle^{\alpha_{m}}, \forall t \geq T_{m}
$$

where

$$
\alpha_{m+1}=p q \alpha_{m}+p+1-\frac{n-1}{2}(p q-1), T_{m}=8^{m} T_{0} .
$$

With help of the claim, we see from (1.10) that

$$
\alpha_{1}=p+1-\frac{n-1}{2}(p q-1)>0, \alpha_{m}>(p q)^{m-1} \alpha_{1}, \forall m \geq 1,
$$

which gives us the desired property $\lim _{m \rightarrow \infty} \alpha_{m}=\infty$ and completes the proof.

It remains to prove the claim, for which we use induction. Assuming that for some $k \geq 0$, we have (5.3) for any $m \leq k$, then by (5.2) we obtain

$$
H^{\prime}(t) \geq C A_{k}^{q}\langle t\rangle^{-\frac{n-1}{2}(q-1)+q \alpha_{k}}, \forall t \geq T_{k} .
$$

As $\frac{n-1}{2}(q-1)<1$ by $(1.10)$, we have $-\frac{n-1}{2}(q-1)+q \alpha_{k}>-1$ and

$$
H(t) \geq H\left(T_{k}\right)+C A_{k}^{q} \int_{T_{k}}^{t}\langle s\rangle^{-\frac{n-1}{2}(q-1)+q \alpha_{k}} \mathrm{~d} s \geq \tilde{C}\langle t\rangle^{-\frac{n-1}{2}(q-1)+1+q \alpha_{k}}
$$

for any $t \geq 2 T_{k}$. Plugging the lower bound (5.5) to the ordinary differential inequality for $G$ in (5.2), we get

$$
G^{\prime \prime}(t)+2 G^{\prime}(t) \geq C \tilde{C}^{p}\langle t\rangle^{-\frac{n-1}{2}(p-1)+p\left(-\frac{n-1}{2}(q-1)+1+q \alpha_{k}\right)}
$$

for all $t \geq 2 T_{k}$, which, by using the multiplier $e^{2 t}$ and integration, yields

$$
G^{\prime}(t) e^{2 t} \geq C\langle t\rangle^{-\frac{n-1}{2}(p q-1)+p+p q \alpha_{k}} e^{2 t}
$$


for any $t \geq 4 T_{k}$. Recall that $p-\frac{n-1}{2}(p q-1)>-1$ by (1.10), we have

$$
G(t) \geq G\left(4 T_{k}\right)+C \int_{4 T_{k}}^{t}\langle s\rangle^{-\frac{n-1}{2}(p q-1)+p+p q \alpha_{k}} d s \geq \tilde{C}\langle t\rangle^{-\frac{n-1}{2}(p q-1)+p+1+p q \alpha_{k}}
$$

for all $t \geq 8 T_{k}$, which gives us (5.3) with $m=k+1$ and completes the proof of the claim.

\subsection{Proof of Lemma 5.3.}

First of all we want to simplify the system (5.2). For any $t \geq 0$, we have

$$
\begin{aligned}
H(t)\left(G^{\prime}(t)+2 G(t)\right)= & \int_{0}^{t} H^{\prime}(s)\left(G^{\prime}(s)+2 G(s)\right) \mathrm{d} s \\
& +\int_{0}^{t} H(s)\left(G^{\prime \prime}(s)+2 G^{\prime}(s)\right) \mathrm{d} s+H(0)\left(G^{\prime}(0)+2 G(0)\right) \\
\geq & C \int_{0}^{t}\langle s\rangle^{-\frac{n-1}{2}(q-1)} G(s)^{q}\left(G^{\prime}(s)+2 G(s)\right) \mathrm{d} s \\
\geq & C\langle t\rangle^{-\frac{n-1}{2}(q-1)} \int_{0}^{t} G(s)^{q} G^{\prime}(s) \mathrm{d} s \\
\geq & \tilde{C}\langle t\rangle^{-\frac{n-1}{2}(q-1)}\left(G^{q+1}(t)-G^{q+1}(0)\right) .
\end{aligned}
$$

Similarly, we have

$$
\left(G^{\prime}(t)+2 G(t)\right)^{p}\left(G^{\prime \prime}(t)+2 G^{\prime}(t)\right) \geq C\langle t\rangle^{-\frac{n-1}{2}(p-1)}\left(H(t)\left(G^{\prime}(t)+2 G(t)\right)\right)^{p} .
$$

Gluing together the above two inequalities, we obtain

$$
\frac{d}{d t}\left(G^{\prime}(t)+2 G(t)\right)^{p+1} \geq C\langle t\rangle^{-\frac{n-1}{2}(p q-1)}\left(G^{q+1}(t)-G^{q+1}(0)\right)^{p},
$$

which gives us

$$
\left(G^{\prime}(t)+2 G(t)\right)^{p+1} \geq C\langle t\rangle^{-\frac{n-1}{2}(p q-1)} \int_{0}^{t}\left(G^{q+1}(s)-G^{q+1}(0)\right)^{p} \mathrm{~d} s
$$

for any $t>0$. Here, since $G, G^{\prime}>0$ for $t>0$ and $G^{\prime}+2 G, G$ are monotonically increasing to infinity, we have

$$
\begin{aligned}
\int_{0}^{t}\left(G^{q+1}(s)-G^{q+1}(0)\right)^{p} \mathrm{~d} s & \geq C \int_{0}^{t}\left(G^{q+1}(s)-G^{q+1}(0)\right)^{p} \frac{\left(G(s)^{q+1}\right)^{\prime}}{\left(G^{\prime}(s)+2 G(s)\right) G(s)^{q}} \mathrm{~d} s \\
& \geq C \frac{\int_{0}^{t}\left(G^{q+1}(s)-G^{q+1}(0)\right)^{p}\left(G(s)^{q+1}\right)^{\prime} \mathrm{d} s}{\left(G^{\prime}(t)+2 G(t)\right) G(t)^{q}} \\
& =\frac{C\left(G^{q+1}(t)-G^{q+1}(0)\right)^{p+1}}{(p+1)\left(G^{\prime}(t)+2 G(t)\right) G(t)^{q}} .
\end{aligned}
$$

Plugging it into (5.6), we obtain

$$
\left(G^{\prime}(t)+2 G(t)\right)^{p+2} \geq C\langle t\rangle^{-\frac{n-1}{2}(p q-1)} \frac{\left(G^{q+1}(t)-G^{q+1}(0)\right)^{p+1}}{G(t)^{q}} .
$$

Choosing a $\tilde{T}_{1}>0$ such that $G\left(\tilde{T}_{1}\right)^{q+1} \geq 2 G(0)^{q+1}$, we have for any $t>\tilde{T}_{1}$

$$
\left(G^{\prime}(t)+2 G(t)\right)^{p+2} \geq C\langle t\rangle^{-\frac{n-1}{2}(p q-1)} G(t)^{p q+p+1} .
$$


As $p q+p+1>p+2$, there exists a $\delta>0$ such that $p q+p+1>(p+2)(1+\delta)+\delta$. Let $M=\frac{n-1}{2}(p q-1) / \delta$ in Lemma 5.2 , we see that there are $\tilde{T}_{2} \geq \tilde{T}_{1}$ and $C>0$ such that

$$
G^{\prime}(t)+2 G(t) \geq C G(t)^{1+\delta},
$$

for any $t \geq \tilde{T}_{2}$. Moreover we can always take a $\tilde{T}_{3} \geq \tilde{T}_{2}$ such that $C G(t)^{1+\delta}>4 G(t)$ for all $t \geq \tilde{T}_{3}$, and so we arrived at the desired ordinary differential inequality

$$
G^{\prime}(t) \geq \frac{C}{2} G(t)^{1+\delta}, G\left(\tilde{T}_{3}\right)>0,
$$

for any $t \geq \tilde{T}_{3}$, which blows up in finite time. This completes the proof.

Acknowledgment. The authors would like to thank the anonymous referee for the careful reading and valuable comments. This work was supported by NSFC 11671353. The third author was supported in part by National Support Program for Young Top-Notch Talents.

\section{REFERENCES}

[1] Rentaro Agemi, Yuki Kurokawa, and Hiroyuki Takamura. Critical curve for $p-q$ systems of nonlinear wave equations in three space dimensions. J. Differential Equations, 167(1):87-133, 2000.

[2] Daniele Del Santo, Vladimir Georgiev, and Enzo Mitidieri. Global existence of the solutions and formation of singularities for a class of hyperbolic systems. In Geometrical optics and related topics (Cortona, 1996), volume 32 of Progr. Nonlinear Differential Equations Appl., pages 117-140. Birkhäuser Boston, Boston, MA, 1997.

[3] Keng Deng. Blow-up of solutions of some nonlinear hyperbolic systems. Rocky Mountain J. Math., 29(3):807-820, 1999.

[4] Lawrence C. Evans. Partial differential equations, volume 19 of Graduate Studies in Mathematics. American Mathematical Society, Providence, RI, second edition, 2010.

[5] Vladimir Georgiev, Hans Lindblad, and Christopher D. Sogge. Weighted Strichartz estimates and global existence for semilinear wave equations. Amer. J. Math., 119(6):1291-1319, 1997.

[6] Wei Han and Yi Zhou. Blow up for some semilinear wave equations in multi-space dimensions. Communications in Partial Differential Equations, 39(4):651-665, 2014.

[7] Kunio Hidano and Kimitoshi Tsutaya. Global existence and asymptotic behavior of solutions for nonlinear wave equations. Indiana Univ. Math. J., 44(4):1273-1305, 1995.

[8] Kunio Hidano, Chengbo Wang, and Kazuyoshi Yokoyama. The Glassey conjecture with radially symmetric data. J. Math. Pures Appl. (9), 98(5):518-541, 2012.

[9] Kunio Hidano, Chengbo Wang, and Kazuyoshi Yokoyama. Combined effects of two nonlinearities in lifespan of small solutions to semi-linear wave equations. Math. Ann., 366(1-2):667-694, 2016.

[10] Kunio Hidano and Kazuyoshi Yokoyama. Life span of small solutions to a system of wave equations. Nonlinear Anal., 139:106-130, 2016.

[11] Jin-Cheng Jiang, Chengbo Wang, and Xin Yu. Generalized and weighted Strichartz estimates. Commun. Pure Appl. Anal., 11(5):1723-1752, 2012.

[12] S. Klainerman. Remarks on the global Sobolev inequalities in the Minkowski space $\mathbf{R}^{n+1}$. Comm. Pure Appl. Math., 40(1):111-117, 1987.

[13] Hideo Kubo, Kôji Kubota, and Hideaki Sunagawa. Large time behavior of solutions to semilinear systems of wave equations. Math. Ann., 335(2):435-478, 2006.

[14] Hans Lindblad and Christopher D. Sogge. Long-time existence for small amplitude semilinear wave equations. Amer. J. Math., 118(5):1047-1135, 1996.

[15] Kyouhei Wakasa Masahiro Ikeda, Motohiro Sobajima. Test function method for blow-up phenomena of semilinear wave equations and their weakly coupled systems. arXiv:1807.03937.

[16] Jason Metcalfe and Chengbo Wang. The Strauss conjecture on asymptotically flat spacetimes. SIAM J. Math. Anal., 49(6):4579-4594, 2017. 
[17] Chengbo Wang and Xin Yu. Recent works on the Strauss conjecture. In Recent advances in harmonic analysis and partial differential equations, volume 581 of Contemp. Math., pages 235-256. Amer. Math. Soc., Providence, RI, 2012.

[18] Borislav T. Yordanov and Qi S. Zhang. Finite time blow up for critical wave equations in high dimensions. J. Funct. Anal., 231(2):361-374, 2006.

[19] Yi Zhou. Life span of classical solutions to $\square u=|u|^{p}$ in two space dimensions. Chinese Ann. Math. Ser. B, 14(2):225-236, 1993. A Chinese summary appears in Chinese Ann. Math. Ser. A 14 (1993), no. 3, 391-392.

[20] Yi Zhou and Wei Han. Blow-up of solutions to semilinear wave equations with variable coefficients and boundary. J. Math. Anal. Appl., 374(2):585-601, 2011.

School of Mathematical Sciences, Zhejiang University, Hangzhou 310027,P.R.China E-mail address: daiw16@zju.edu.cn

School of Mathematical Sciences, Zhejiang University, Hangzhou 310027,P.R.China E-mail address: dyf@zju.edu.cn

School of Mathematical Sciences, Zhejiang University, Hangzhou 310027,P.R.China E-mail address: wangcbo@zju.edu.cn

$U R L:$ http://www.math.zju.edu.cn/wang 